\title{
Large lateral dermoid cyst mimicking plunging ranula: A case report and intraoral approach to surgical management
}

\author{
Alhassan Emil Abdulai, Dominic Avogo, Isaac Kwasi Nuamah, \\ Henry Baddoo, Richard Kwasi Gyasi
}

\begin{abstract}
Introduction: Dermoid cysts are benign cystic teratomas with epithelial lined cavities, bearing hair follicles, hair, sebaceous glands, sweat glands, and occasionally tissues derived from the mesoderm. Dermoid cysts of the floor of the mouth are relatively rare but when they occur, are commonly restricted to one anatomic zone and in the midline region. They are treated surgically. Case Report: We present a case of a lateral dermoid cyst mimicking a plunging ranula extending from the upper part of the neck, on the right side, upwards into the sub-mandibular region and via a hiatus in the mylohyoid muscle into the floor of the mouth, in a 24-year-old Ghanaian male. Conclusion: The treatment of dermoid cysts of the floor of the mouth is surgical, using either an intraoral, extra oral or both approaches depending on the localization
\end{abstract}

\footnotetext{
Alhassan Emil Abdulai ${ }^{1}$, Dominic Avogo ${ }^{2}$, Isaac Kwasi Nuamah $^{3}$, Henry Baddoo ${ }^{4}$, Richard Kwasi Gyasi ${ }^{5}$

Affiliations: ${ }^{1}$ BDS, FWACS, FGCS, Department of Oral and Maxillofacial Surgery, University of Ghana Dental School, Korle Bu Teaching Hospital, Accra, Ghana; ${ }^{2} \mathrm{BDS}$, FGCS, Department of Oral and Maxillofacial Surgery, University of Ghana Dental School, Korle Bu Teaching Hospital, Accra, Ghana; ${ }^{3} \mathrm{BDS}, \mathrm{MB}, \mathrm{ChB}$, FDS, FGCS, Department of Oral and Maxillofacial Surgery, University of Ghana Dental School, Korle Bu Teaching Hospital, Accra, Ghana; ${ }^{4} \mathrm{MB}, \mathrm{ChB}, \mathrm{FRCA}$, FRCS Ed. FWACS, Department of Anaesthesia, University of Ghana Medical School, Korle Bu Teaching Hospital, Accra, Ghana; ${ }^{5} \mathrm{MB}, \mathrm{ChB}$, FWACPath, FGCS, Department of Pathology, University of Ghana Medical School, Korle Bu Teaching Hospital, Accra, Ghana.

Corresponding Author: Alhassan Emil Abdulai, P.O. Box C859, Cantonments.Accra, Ghana; Ph: +233 244 387707; Email: aemilabdulai@yahoo.com
}

Received: 15 December 2012

Accepted: 31 May 2013

Published: 01 June 2014 and size of the mass. An intraoral approach to a successful surgical management of this very large cyst is also described. This is a rare, interesting case of a lateral dermoid cyst, unusually transcending three anatomical spaces, in an ethnic Ghanaian with a significant portion below the mylohyoid that was successfully managed using a wholly intraoral surgical approach.

Keywords: Dermoid cyst, Intraoral,Submandibular

\section{How to cite this article}

Abdulai AE, Avogo D, Nuamah IK, Baddoo H, Gyasi RK. Large lateral dermoid cyst mimicking plunging ranula: A case report and intraoral approach to surgical management. Int $\mathrm{J}$ Case Rep Images 2014;5(6):408-412.

doi:10.5348/ijcri-201477-CR-10388

\section{INTRODUCTION}

Dermoid and epidermoid cysts are benign cystic teratomas with epithelial lined cavities, bearing hair follicles, hair, sebaceous glands, sweat glands, and occasionally tissues derived from the mesoderm $[1$, 2]. They usually present early in life as asymptomatic masses with slow and progressive growth, and may reach large sizes, involve more than one anatomical area and or may abut the hyoid bone when in the neck [1]. They are encountered throughout the body and are normally found in the midline areas [2]. About $6.9 \%$ of all dermoid cysts occur in the head and neck region, and are commonly located in the eye brow area where they are well-known as angular dermoid. Approximately 1.6-1.8\% occur within the oral cavity [1]. The floor of the mouth is their preferred location, and the sublingual, sub mental, or laterally between the hyoglossus and mylohyoid 
muscles are locations of occurrence in descending order of frequency [1-4]. Plunging dermoid cysts are extremely rare $[1,2]$; and so are lateral dermoid cysts [5]. They have both often posed some diagnostic confusion when they occur $[1,5]$. The treatment of dermoid cysts of the floor of the mouth is surgical, using either an intraoral, extraoral or both approaches depending on the localization and size of the mass [4]. We present a case of large lateral dermoid cyst that had been present since birth with an oral, submandibular as well as upper cervical component, in a 24-year-old male patient, and discuss an intraoral approach to its successful surgical management.

\section{CASE REPORT}

A 24-year-old Ghanaian male was presented to the Maxillo-facial unit of the Korle Bu Teaching Hospital, Accra, with a swelling of the upper part of the neck, on the right side, extending upwards into the right submandibular region and into the mouth below his tongue. He first noticed the swelling himself at the age of 15, though according to his parents, it had been present since birth as a small lump in the upper part of the neck below the angle of the mandible and has increased progressively over the years. Initially it was painless, but had recently begun to hurt at mealtimes, hence the reason for seeking medical attention.

The swelling was located in the right submandibular space (Figure 1). The swelling extended downwards into the upper cervical area, and upwards into the region of the floor of the mouth on the same side. It was doughy, not tender, non-pulsating, not fixed to neighboring structures and measured about 7-9 $\mathrm{cm}$ in diameter. It was reducible and moved with swallowing. The overlying skin had a smooth surface, and there was no lymphadenopathy. Intraorally, the swelling spanned, asymmetrically below the mucosa, from the second lower right molar region across the floor of the mouth to the lower incisor region on the left. The oral mucosa appeared normal albeit with prominent and tortuous vessels, giving an appearance resembling a 'frog's belly' reminiscent of a ranula (Figure 2).

Hematological investigations and chest X-ray were within normal limits. Computed tomography (CT) scan of the lesion across the horizontal plane resembled an inverted map of Africa (Figure 3). It showed an anteriorlateral cervical cystic swelling, extending from the occlusal surfaces of teeth to the level of the thyroid cartilages. The cystic wall appeared relatively thicker posteriorly. The pharynx was slightly narrowed and deviated to the left. Differential clinical diagnosis at this stage included a plunging ranula and a dermoid/epidermoid cyst.

The patient underwent surgery under general anesthesia with nasotracheal intubation. Gentle manual external pressure on the lesion in the submandibular region made it prominent in the sublingual region. A horizontal incision was then made lateral and parallel to the right Wharton's duct through the mucosa in the

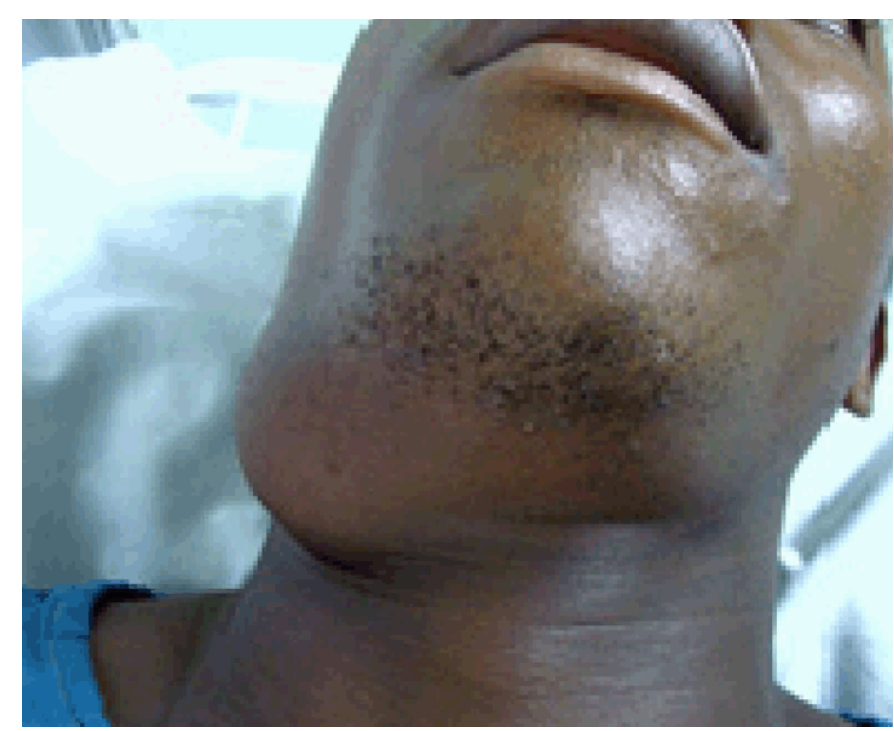

Figure 1: Photograph of patient showing right submandibular swelling (preoperative) at Clinical presentation.

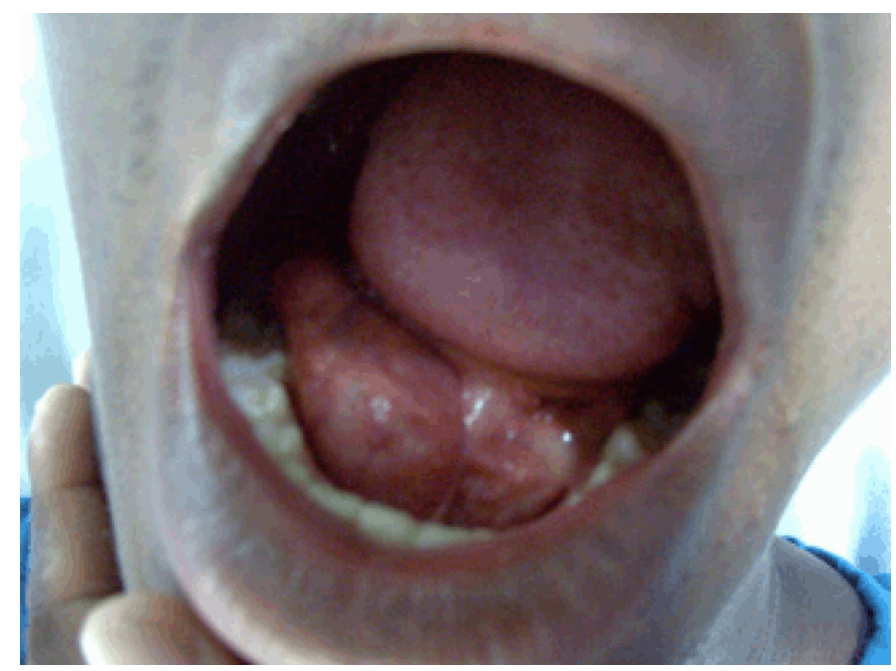

Figure 2: Photograph of patient showing 'frog belly' look of the lesion reminiscent of a ranula.

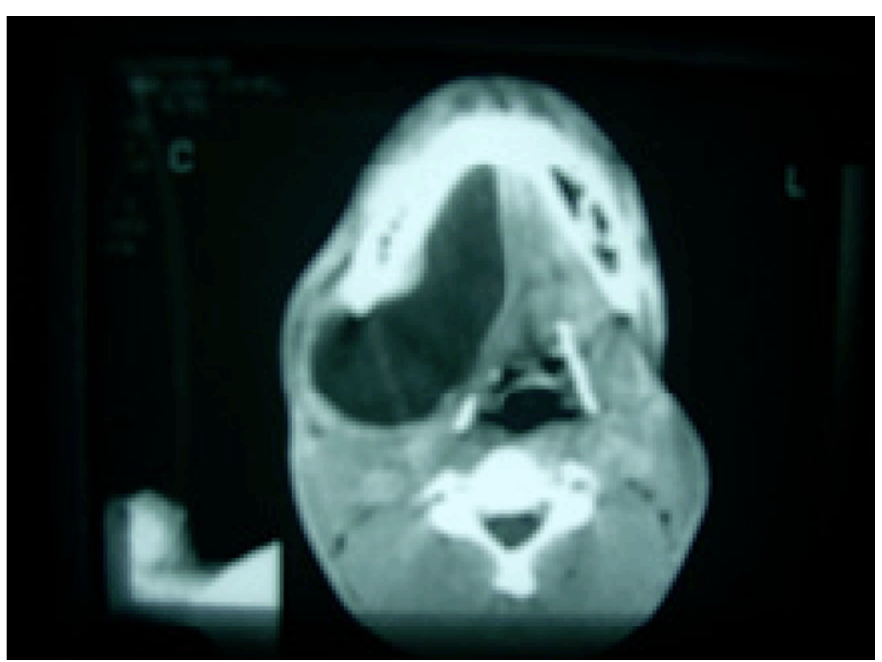

Figure 3: Computed tomographic scan of the lesion across the horizontal plane showing the extent of the laterally positioned cyst. Note similarity of the shadow to an inverted map of Africa. 
lingual sulcus, from the mesial end of the first molar, and carried anteriorly to stop just short of the lingual frenum. Special care was taken to avoid damage to the Wharton's duct or lingual nerve. The mucosa was then reflected to expose the cystic lesion. Aspiration of pale yellow copious fluid from the cyst at this stage was very helpful in ruling out ranula.

The cyst was partially exposed, and to enhance delivery of the rather large cyst through the intraoral approach, a window was made into it and most of its contents, consisting of a rather thick creamy homogenous paste, evacuated. Stay sutures were inserted through the wall of the cyst, at the edges of the window; to enable upward traction, while blunt dissection was carried out at its periphery to free it (Figure 4). Final delivery was aided by the insertion of the middle finger at the periphery of the cyst, downward unto the mylohyoid muscle, and dilating the hiatus created through the muscle, while at the same time, freeing the deeper attachment of the cyst and gently retracting the cyst upwards.

Minimal hemorrhage was encountered; and the wound was closed in layers without placing a drain. A crepe bandage dressing was applied over the head to provide pressure against the wound, and prevent surgical emphysema and postoperative swelling. The procedure was well tolerated, and the patient discharged after five days of hospitalization. At fourth week postoperative follow-up, the patient appeared in excellent condition.

Histopathological examination with hematoxylineosin staining revealed a cystic lesion with a stratified squamous epithelium lining; with sebaceous glands and hair follicle in wall, and a fibrovascular connective tissue capsule covering the cystic lumen consistent with dermoid cyst (Figure 5).

\section{DISCUSSION}

Dermoid and epidermoid cysts are rare in the head and neck region (6.9\%). They are even rarer in the oral

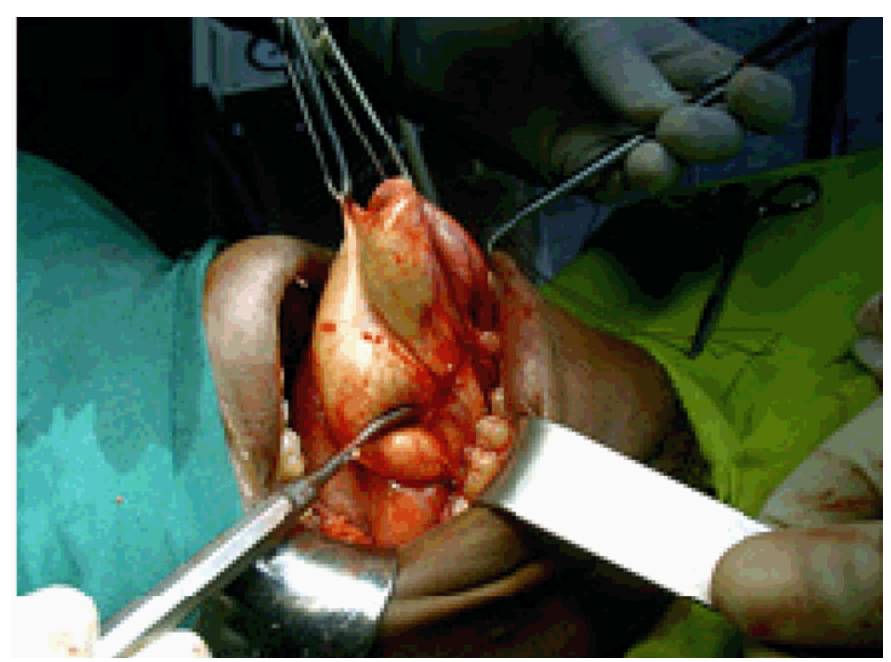

Figure 4: Intra-operative photograph showing "Stay sutures" in place to aid in traction of the cyst. Note minimal bleeding.

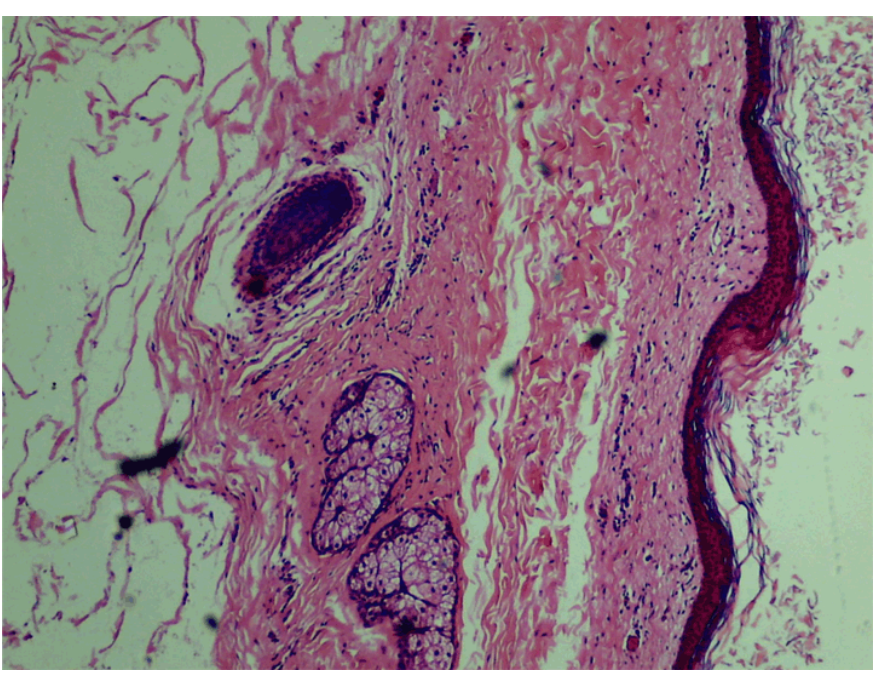

Figure 5: Photomicrograph of lesion showing squamous epithelial lining with sebaceous glands and hair follicle in wall, consistent with dermoid cyst (H\&E stain, X400).

cavity (1.6-1.8\%) mostly occurring in the sublingual region [1]. The differential diagnosis of a sublingual lesion includes: ranula, lymphatic malformation, dermoid cyst, epidermoid cyst, heterotopic gastrointestinal cyst and duplication foregut cyst [1]. Bimanual palpation and conventional radiography are not always sufficient in making differential diagnoses. In these cases, it is necessary to use ultrasonography, computed tomography, or magnetic resonance imaging scans together with cytological examination by fine-needle aspiration biopsy [6]. Ultrasonography represents the first choice of imaging technique because it is reliable, economical, and without X-ray exposure. Computed tomography scan and magnetic resonance imaging scan allow more precise localization of the lesion in relationship to geniohyoid and mylohyoid muscles, and they also enable the surgeon to choose the most appropriate surgical approach, especially for very large lesions [6]. In our case, the CT scan image which the patient presented to us from the referring physician offered such clarity that we did not carry out any further investigations. Initial attempt to aspirate any fluid from the lesion at the chair side was unsuccessful. The cheesy content of the lesion was confirmed at surgery, perhaps explaining our unsuccessful attempt to aspirate at chair side; another reason may be that the fluid part of the lesion was superiorly located from the submandibular percutaneous site of our attempted aspiration.

Ranula, especially the plunging type is one sublingual lesion notorious for extending into the neck. Its management has been discussed by Patel et al. who suggested that definitive treatment yielding lowest recurrence and complication rates for all ranulas are trans-oral excision of the ipsilateral sublingual gland with ranula evacuation [7]. A few cases of dermoid cysts have been reported to involve more than one anatomic area, though, rather than lateral as reported here, these 
were located in the midline $[1,8]$. The best surgical approach to a mass that involves two or more anatomical locations has often been debatable. Bitar and Kumar used the cervical approach, solely because the cervical component of the cyst they encountered was larger [9]. Al-Khayat and Kenyon, on the other hand, reported a combined approach (intraoral and cervical) to excise a "plunging" dermoid cyst, which was extending from the sublingual space to the left submandibular area past the lateral border of the mylohyoid muscle [8]. Di Francesco et al. avoided additional skin incision by aspirating the content of the cyst before excising it through the intra oral approach [9].

In our case, the cyst was herniating through the lateral portion of the mylohyoid muscle on the right into the submandibular and upper cervical areas, and was easy to reduce. This among others influenced our choice of intraoral approach. Using this method to treat the cyst and prevent external scars restricted our use of any extraoral drains. We addressed the issue of reducing the spaces where hematoma could form by placing a crepe bandage dressing round the head and over the chin to provide pressure against the wound, in other to prevent surgical emphysema and reduce postoperative swelling using its pressure effect. Also widely separated interrupted sutures allowed drainage in the mouth. There is always a risk of keloid formation during wound scarification, especially in Negroid patients, leading to unsightly aesthetics. Hence whenever possible, the intraoral approach is to be preferred. The challenge posed in our case stemmed from the lateral location of the cyst that necessitated making an incision in the lingual sulcus, with the attendant risks of causing damage to either the Wharton's duct, lingual nerve or both. Fortunately, no such hazard was encountered. Reducing the cyst by maintaining gentle manual external pressure, made the lesion prominent sublingually, thereby requiring only an incision through the overlying mucosa to expose the thick cystic lining, and avoid any collateral damage to vital structures in the region. We employed a method akin to that used by Di Francesco et al. to decompress the cyst before excising it through the intraoral approach [10]. A window was made into the cyst that allowed evacuation of some of its contents (thick creamy cheesy putty-like material), thereby decompressing the cyst and making it easier to extract through the hiatus in the mylohyoid muscle. This also enabled the examination of the contents to confirm or refute the diagnosis of a dermoid cyst, as suggested by Sullivan et al. [10].

\section{CONCLUSION}

This is a rare case of a lateral dermoid cyst, unusually transcending three anatomical spaces, in an ethnic Ghanaian with a significant portion below the mylohyoid and it was successfully managed using a wholly intra oral surgical approach.

\section{Author Contributions}

Alhassan Emil Abdulai - Substantial contributions to conception and design, Acquisition of data, Analysis and interpretation of data, Drafting the article, Revising it critically for important intellectual content, Final approval of the version to be published

Dominic Avogo - Analysis and interpretation of data, Revising it critically for important intellectual content, Final approval of the version to be published

Isaac Kwasi Nuamah - Analysis and interpretation of data, Revising it critically for important intellectual content, Final approval of the version to be published

Henry Baddoo - Analysis and interpretation of data, Revising it critically for important intellectual content, Final approval of the version to be published

Richard Kwasi Gyasi - Analysis and interpretation of data, Revising it critically for important intellectual content, Final approval of the version to be published

\section{Guarantor}

The corresponding author is the guarantor of submission.

\section{Conflict of Interest}

Authors declare no conflict of interest.

\section{Copyright}

(C) 2014 Alhassan Emil Abdulai et al. This article is distributed under the terms of Creative Commons Attribution License which permits unrestricted use, distribution and reproduction in any medium provided the original author(s) and original publisher are properly credited. Please see the copyright policy on the journal website for more information.

\section{REFERENCES}

1. Bitar MA, Kumar S. Plunging congenital epidermoid cyst of the oral cavity. Eur Arch Otorhinolaryngol 2003;260(4):223-5.

2. Mathews J, Lancaster J, O’Sullivan G. True lateral dermoid cyst of the floor of the mouth. J Laryngol Otol 2001;115(4):333-5.

3. Kandogan T, Koç M, Vardar E, Selek E, Sezgin O. Sublingual epidermoid cyst: a case report. Journal of Medical Case Reports 2007;1:87.

4. Ohta N, Watanabe T, Ito T, et al. A Case of Sublingual Dermoid Cyst: Extending the Limits of the Oral Approach. Case Rep Otolaryngol 2012;2012:634949.

5. Graham RM, Thomson EF, Woodwards RT, Sloan P. Lateral dermoid cyst. Br J Oral Maxillofac Surg 2008;46(2):131-2.

6. Walstad WR, Solomon JM, Schow SR, Ochs MW. Midline cystic lesion of the floor of the mouth. J Oral Maxillofac Surg 1998;56(1):70-4.

7. Patel MR, Deal AM, Shockley WW. Oral and plunging ranulas: What is the most effective treatment? Laryngoscope 2009;119(8):1501-9. 
8. Al-Khayat M, Kenyon GS. Midline sublingual dermoid cyst. J Laryngol Otol 1990;104(7):578-80.

9. Di Francesco A, Chiapasco M, Biglioli F, Ancona D. Intraoral approach to large dermoid cysts of the floor of the mouth: a technical note. Int J Oral Maxillofac Surg 1995 Jun;24(3):233-5.

10. Sullivan DP, Liberatore LA, April MM, Sasson J, Ward RF. Epidermal inclusion cyst versus thyroglossal duct cyst: sistrunk or not? Ann Otol Rhinol Laryngol 2001 Apr;110(4):340-4.

Access full text article on other devices

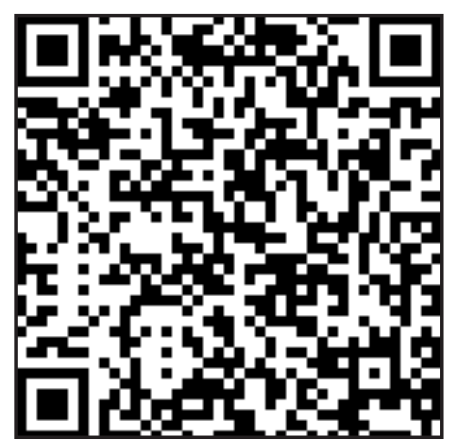

Access PDF of article on other devices

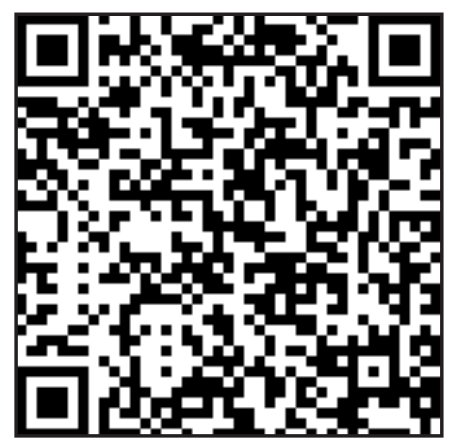

